\title{
Demo: STC-CAM1, IR-Visual Based Smart Camera System
}

\author{
Muhammad Imran, Mattias O’Nils, Victor Kardeby, Huma Munir \\ Mid Sweden University \\ Holmgatan 10, SE-85170 \\ Sundsvall, Sweden \\ \{muhammad.imran, mattias.onils, victor.kardeby\}@miun.se; humu1200@student.miun.se
}

\begin{abstract}
Safety-critical applications require robust and real-time surveillance. For such applications, a vision sensor alone can give false positive results because of poor lighting conditions, occlusion, or different weather conditions. In this work, a visual sensor is complemented by an infrared thermal sensor which makes the system more resilient in unfavorable situations. In the proposed camera architecture, initial data intensive tasks are performed locally on the sensor node and then compressed data is transmitted to a client device where remaining vision tasks are performed. The proposed camera architecture is demonstrated as a proof-ofconcept and it offers a generic architecture with better surveillance while only performing low complexity computations on the resource constrained devices.
\end{abstract}

\section{Keywords}

Wireless smart camera, Infrared, Thermal, Architecture, Wireless vision sensor node, Internet-of-Things.

\section{INTRODUCTION}

Recent advances in the field of electronics, optics and communication are enabling the development of wireless smart camera systems which are expected to have embedded processing for handling large amount of data [1][2]. These advancements facilitate development of stand-alone, scalable, easy-to-integrate, low cost, and low power solutions for a number of potential applications. Typical applications include surveillance for safetycritical and hard real-time systems, health care, machine vision and smart home systems [3]-[5].

This paper focuses on safety critical applications, where visual sensors alone are not enough for satisfactory surveillance, because vision sensors are sensitive to visible range of the electromagnetic spectrum. Therefore for better spectral resolution, vision sensors can be complemented with beyond-vision-range sensors such as infrared i.e. thermal sensors. The infrared sensors generate thermographic images by sensing the infrared radiation, emitted from the objects with an absolute temperature greater than zero. These two image sensors, sensitive to broader range of electromagnetic spectrum offer better information of the situation when the sensed data is fused together. In relation to this, there are three prominent fusion methods applied at different processing levels [3][4], namely pixel, feature, and decision fusing. These approaches are often processed on powerful personal computers, which simplifies the problem because porting a concept to embedded platforms will arise in new set of challenges, for example

Permission to make digital or hard copies of part or all of this work for personal or classroom use is granted without fee provided that copies are not made or distributed for profit or commercial advantage and that copies bear this notice and the full citation on the first page. Copyrights for third-party components of this work must be honored. For all other uses, contact the Owner/Author.

Copyright is held by the owner/author(s)

ICDSC ' 15 , September 08-11, 2015, Seville, Spain

ACM 978-1-4503-3681-9/15/09.

http://dx.doi.org/10.1145/2789116.2802649 resources constraints and design complexity [1]. In this paper, we are proposing a visual and infrared based smart camera architecture, which has embedded in-node processing and wireless communication capabilities. Our approach is different from the existing approaches because of the considerations for embedded implementation and a different fusion method.

Compared to the three mentioned fusion approaches, we perform computation on the thermal stream of images and blend processed data with visual stream of images in order to highlight activity regions. This architecture requires frequent transmission of regionof-interest data from thermal sensor and occasional transmission of full size image from visual sensor. The proposed camera architecture is based on bio-inspired approach of partitioning tasks between camera node (infant) and a client device (mature) as shown in Figure 1. This approach helps in relaxing both processing and transmission requirements. It is worth mentioning that in this work, processing on camera node is considered and processing on client is not discussed. The proposed architecture offers better surveillance and results in a generic architecture because of commonly used pre-processing tasks of the surveillance applications.

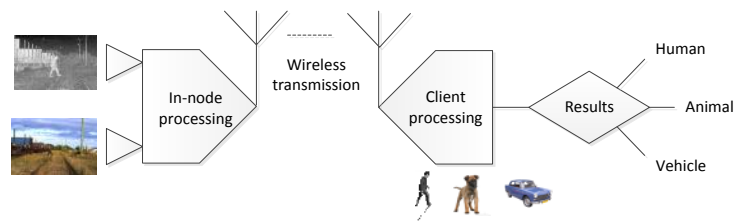

Figure 1. System architecture with intelligence partitioning.

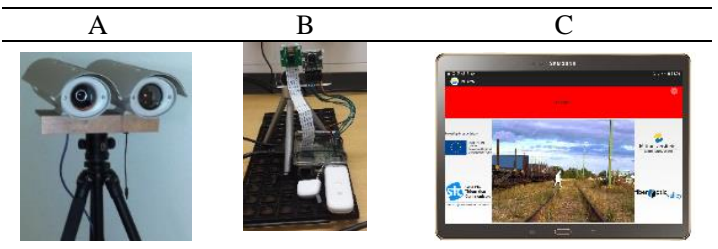

Figure 2. IR-Visual Smart Cameras. (A) Field Setup.

(B) Demo Setup. (C) Client device.

\section{DEMONSTRATOR}

The setup for the field experiments is shown in Figure 2 (a) and a demonstration setup, a smaller variant of the field setup, is shown in Figure 2 (b). A preview of demo can be accessed online [6]. The demonstrator main components include a 5 megapixel Omnivision color image sensor [7], a FLIR one thermal image sensor [8], a Raspberry Pi 2 model B [9], and a ZTE MF823 USB-modem for LTE and HSPA+ [10]. The client device, Samsung Galaxy Tab S, is shown in Figure 2 (c).

\subsection{Scenarios}

The focus of this demonstrator is on scenarios which have a safetycritical nature and real-time requirement. These scenarios include pedestrian detection on roads, rail tracks, people positioning 


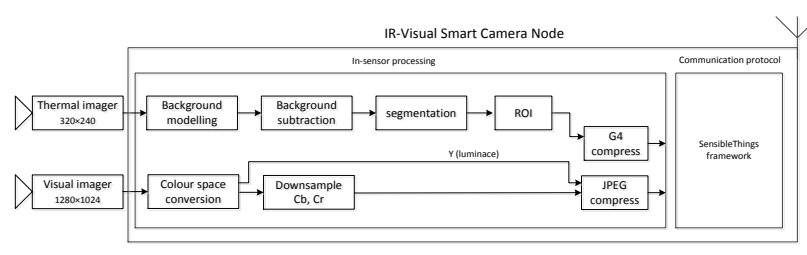

Figure 3. Architecture of proposed IR-Visual Smart Camera.

in endangered places with poor lighting conditions. For such scenarios, the combined processed information of thermal and visual with embedded implementation could meet the demand. The specific test case in the demonstrator is object detection near rail tracks.

\subsection{STC-CAM1 architecture}

STC-CAM1 architecture is shown in Figure 3. In this architecture, data intensive tasks are performed on the camera node whereas the high level tasks e.g., feature extraction, identification and classification are performed on the client device. This makes architecture generic because pre-processing are same for many surveillance applications [5]. STC-CAM1 consists of two modules, in-node processing module and a communication module.

\subsection{In-node processing}

In the proposed architecture, the image processing tasks e.g., background modelling, subtraction, segmentation, Region-ofInterest and ITU-T G4 compression are performed on thermal images. Following this, compressed binary data is transmitted frequently to client in order to monitor the activity in the area of interest. On the visual stream of images, JPEG compression is performed on the luminance $(\mathrm{Y})$ component and on the downscaled color components $(\mathrm{CbCr})$. The color coded images are transmitted occasionally in order to give situational awareness and detailed information. This would result in overall small amount of data from camera node as shown in Table 1 but still having important information of the monitored area. Sample images of the setup are shown in Figure 4. Table 1 shows average amount of data transmitted from the camera node with mentioned transmission frequency.

\section{Table 1. Salient features of the STC-CAM1}

\begin{tabular}{|c|c|c|c|}
\hline Sensor type & Raw data & $\begin{array}{l}\text { Avg. output } \\
\text { data (one } \\
\text { person) }\end{array}$ & $\begin{array}{l}\text { Frequency of } \\
\text { transmission* }\end{array}$ \\
\hline Visual & $3.75 \mathrm{MB}$ & $167 \mathrm{~KB}$ & Occasionally \\
\hline Thermal & $82.5 \mathrm{~KB}$ & 475 Bytes & Frequently \\
\hline
\end{tabular}

\subsection{Communication protocol}

The communication to the client device is handled by SensibleThings platform [11], which is an Internet-of-Things (IoT) networking platform for enabling Peer-to-Peer (P2P) communication between connected things on the Internet. The P2P nature of the communication enables the system to have approximately half latency as compared to traditional cloud services which need to proxy all data through a centralized point [12]. The platform is easy to integrate for different IoT applications and it is compatible with both Android and Raspberry Pi based systems.

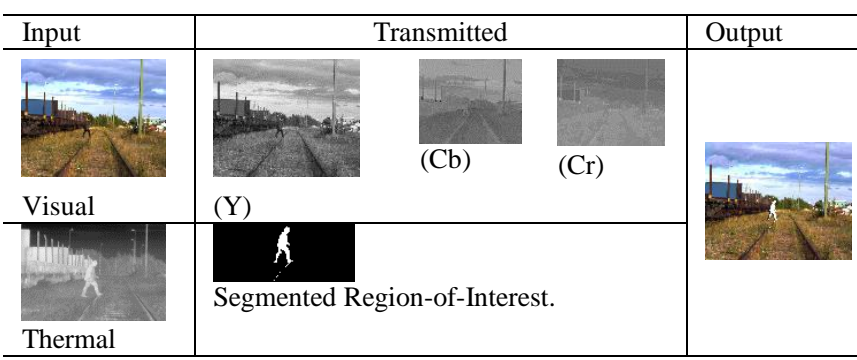

Figure 4. Sample images of STC-CAM1 for different stages. (1) input, (2) transmission and (3) output.

\section{ACKNOWLEDGMENTS}

The authors acknowledge the efforts of Vincent Skerved for assisting in developing the camera platform, and Stefan Forsström for his work with the SensibleThings platform. This research is funded by the Swedish Knowledge Foundation and by the Ericsson Research Foundation.

\section{REFERENCES}

[1] M. Imran, K. Shahzad, N. Ahmad, M. O'Nils, N. Lawal, B. Oelmann, "Energy-Efficient SRAM FPGA-Based Wireless Vision Sensor Node: SENTIOF-CAM," IEEE Transactions on Circuits and Systems for Video Technology, vol.24, no.12, pp.2132,2143, Dec. 2014

[2] Bailey, D. G. 2011. Design for Embedded Image Processing on FPGAs, Asia, John Wiley \& Sons, (2011), pp. 199-231.

[3] S. Ibrahim and M. Wirth, "Visible and IR Data Fusion Technique Using the Contourlet Transform," Int. Conf. Comput. Sci. Eng., vol. 2, pp. 42-47, 2009.

[4] T. Mouats and N. Aouf, "Fusion of thermal and visible images for day/night moving objects detection". Sensor Signal Processing for Defence (SSPD), 2014.

[5] M. Imran, K. Benkrid, K. Khursheed, N. Ahmad, M. A. Waheed, M. O'Nils, N. Lawal, "Analysis and characterization of embedded vision systems for taxonomy formulation", SPIE Real-Time Image and Video Processing, USA, Feb. 2013.

[6] IR-VISUAL STC-CAM1 demo, Available online https://youtu.be/JNEdIpIZ7ow

[7] OmniVision Technologies Inc, Omnivision 5647 sensor, (2013) [online]. Available: www.ovt.com/

[8] FLIR Systems, FLIR ONE. (2015) [online]. Available: http://www.flir.se/

[9] Raspberry Pi Foundation, Raspberry Pi 2 Model B. (2015) [online]. Available online: https://www.raspberrypi.org/

[10] ZTE Corporation, ZTE MF823 USB-modem (2015) [online]. Available: http://www.ztedevice.com/

[11] S. Forsström, V. Kardeby, P. Österberg, and U. Jennehag, "Challenges when Realizing a Fully Distributed Internet-ofThings - How we Created the SensibleThings Platform". In proceedings of 9th IARIA International Conference on Digital Telecommunications, 2014.

[12] V. Kardeby, U. Jennehag, and M. Gidlund, "Power consumption for global information dissemination in the Internet of Things". Tenth Intl. Conf. on Intelligent Sensors, Sensor Networks and Info. Processing (ISSNIP), 2015. 